\title{
Brief Announcement: A Conjecture on Traceability, and a New Class of Traceable Networks
}

\author{
H. B. Acharya ${ }^{1}$, Anil K. Katti ${ }^{1}$, and Mohamed G. Gouda ${ }^{1,2}$ \\ 1 The University of Texas at Austin, USA \\ acharya @cs.utexas.edu, \\ 2 The National Science Foundation, USA
}

\begin{abstract}
The problem of reconstructing the topology of a network, given a set of hop-by-hop traces of packet paths through it, is called 'network tracing'. Unfortunately, there are only a few known classes of networks (trees and odd rings) that are traceable, i.e. that have the property that a unique network topology can be reconstructed from a trace set. Here, we suggest a property that may be the reason such networks are traceable, and use it to identify a new class of traceable networks.
\end{abstract}

\section{Introduction}

Many applications improve performance by using network locality; hence, knowledge of the network topology is important, but not readily available. There have been many attempts to map networks, such as Rocketfuel [2]; these mappers use Traceroute to obtain hop-by-hop paths between known start and end nodes, called terminals, then reconstruct a candidate topology that contains the known paths.

Unfortunately, this approach leads to wildly inaccurate maps. In a trace, every node other than the (known) terminals may refuse to reveal its IP address, so nodes are anonymous; some nodes have many IP addresses (eg. for multihoming) hence there is aliasing; and many nodes and edges do not appear in the trace set at all.

In our previous work [1], we demonstrated that even if we can ensure complete coverage, knowledge of aliases, and consistent routing, a single anonymous or irregular (sometimes-anonymous) node can make it impossible to uniquely reconstruct a general network from a trace set. Networks are traceable (i.e. can be uniquely reconstructed from their trace sets) despite the presence of irregular nodes if the topology of the network is known to be a tree or an odd ring.

In our present work, we make a conjecture as to the property of trees and odd rings that makes them traceable (possible to uniquely identify from a trace set), and report on our progress toward proving the correctness of our conjecture. Using this conjecture, we identify a new class of traceable network. 


\section{The Unique Path Length Conjecture}

We begin by noting that a trace set only reveals the distance between nodes along one path (the path of the trace). But this is disproportionately important for a tree network, where there is exactly one path between any two nodes; the algorithm to trace a tree network identifies the nodes using their distances from two terminals, and if there were many paths between the same two nodes, there would be multiple nodes with the same distances to both terminals.

What of odd rings? In any ring, there are two paths between any two nodes $a$ and $c$; if we take rings with a third node $b$, there is a path from $a$ to $c$ passing through $b$, and a path that does not pass through $b$. In answer, we note that in addition to which terminals it runs between, a trace has another defining characteristic: its length. In an odd ring, there is exactly one path of a given length between any two nodes. (Let $|a b c|$ denote the length of the arc connecting $a$ to $c$ through $b$, and $\left|a b^{\prime} c\right|$ the length of the other arc from $a$ to $c$. In a ring where $|a b c|=\left|a b^{\prime} c\right|$, the total length of a circuit around the ring, i.e. $|a b c|+\left|a b^{\prime} c\right|$, is even - and even rings are known to not be traceable.)

In fact, the algorithm to trace odd rings does make use of this characteristic. In the algorithm, we begin by fixing a terminal node $a$ and marking the nodes of one trace $(a \ldots b)$ randomly around the ring (clockwise or counterclockwise). Next, we place all remaining traces with terminal node $a$ on the ring. For such a trace $(a \ldots c)$, we choose the opposite direction as $(a \ldots b)$ if $|a c|+|a b|+|b c|=$ ring.length, and the same direction as $(a \ldots b)$ otherwise. At the end, all the terminal nodes are placed, and we can fill in any missing labels using the fact that there is only one path of a given length between terminals (so if, say, $x$ is 2 hops from terminal $y$ along the $\operatorname{arc}(y \ldots z)$ of length 5 , this uniquely specifies its position on the ring).

Based on the above discussion, we suggest the following conjecture.

Conjecture $1 A$ network is traceable if, given any two nodes in $N$ and a length $l$, there is no more than one path of length $l$ connecting these nodes in the network.

We are currently investigating the truth of this conjecture. We have found the encouraging partial result that another class of network satisfying the given condition, unicyclic networks in which the length of the cycle is odd, are also traceable (provided that all nodes shared by a tree and the cycle are terminal, and the terminal nodes are regular).

\section{References}

1. H. B. Acharya and M. Gouda. On the hardness of topology inference. In 12th International Conference on Distributed Computing and Networking, January 2011.

2. N. Spring, R. Mahajan, D. Wetherall, and T. Anderson. Measuring isp topologies with rocketfuel. IEEE/ACM Trans. Netw., 12(1):2-16, 2004. 